EPiC Series in Computing
Volume 77, 2021, Pages 115-122
$\begin{aligned} & \text { Proceedings of ISCA 30th International Confer- } \\ & \text { ence on Software Engineering and Data Engineering }\end{aligned}$

\title{
Automated Light System in Smart Home for Elderly and Disabled People Using Mosquitto Server
}

\author{
Paniti Netinant ${ }^{1}$, Kawinna Sakornthai ${ }^{1}$ and Meennapa Rukhiran ${ }^{2 *}$ \\ ${ }^{1}$ College of Digital Innovation Technology, Rangsit University, Thailand \\ ${ }^{2}$ Faculty of Social Technology, Rajamangala University of Technology Tawan-OK, Thailand \\ paniti.n@rsu.ac.th, misskawinnalgmail.com, \\ meennapa_ruermutto.ac.th
}

\begin{abstract}
Internet of things to applications have the potential to improve the quality of life, especially for elderly and disabled people. A smart home has been a beneficial system for elderly and disabled people. However, there are many innovative gaps to design, develop, install, and plugins for improving an intelligent home system. Basic activities should be initially concerned, such as electronic control systems, human movement detection, and environment monitor system. There are many issues in designing an intelligent home system, such as what the devices need to integrate into the system and designing a thin system. This paper explores the design and development of the automated light system in a smart home using the Internet of things and Mosquitto server. This research aims to design the workflow overview of the automatic light system based on sensors, devices, components, and servers. The system achieves the light control manually and automatically. MQTT server accomplishes to control and modify any devices in the IoT network system flexibly. The system's functionalities achieve, and the motion sensor capability is a concern of the sensing range covering the area.
\end{abstract}

\section{Introduction}

Internet of Things has now become an essential mechanism of human lives over the years. In the intervening period, the intelligent systems have a remarkable growth of industrial embedded computers to the smart city. Through the Internet of Things (IoT) era, the traditional approaches are progressively changing and converging to automated home systems. Although innovative home

\footnotetext{
${ }^{*}$ Corresponding author
} 
technologies are presently being installed, they are limited to particular applications for unique care users such as early, disable, and injured people. Our inspiring purposes are to design an automated light system to ensure interoperability for early and disable people. The lights can turn on/off automatically without any assistance [1]. The elderly feel comfortable living in their smart house [2].

Many studies have applied IoTs as an activate service to develop smart houses in many areas. An intelligent concept of assisting independent living has been proposed by [3]. The goals and targets of the philosophical system are to detect [4], monitor, analysis, and treat to ensure that people who live independently enable to live their lives comfortably and safely. The particular elements of the IoT are included medical detection such as blood pressure, heart rate, and body weight, and home management such as TV, light, and mobile. Ainane, Ouzzif, and Bouragba [5] have mainly studied a sensor-based smart home such as camera sensors and mouthpieces. The K-NN algorithm considers classifying an overall accuracy of $51 \%$ for sensor values prediction. IoT platform provides main features for electronic home connection, security enhances, smart devices monitor and control, and activate automation [6]. Moreover, home equipment and smart devices such as smartphones, motion sensors, TVs, Bluetooth trackers, surveillance cameras, and wearable technology support data collection using Multi-Agent System. The system enables recording data from many devices using cloud technology. Block diagram of typical home automation system [7] represents supporting home technological designs such as lighting, IR camera, innovative shadows, air conditioning, and gas sensor. For example, programming language to develop a door monitoring system [8], Python language, and OpenCV library can implement using Raspberry PI [9]. IoT technology of face recognition and voice command are used to transform the regular door into an automatic gate with ZigBee Modulation. The result can reduce humane efforts, save electricity, and achieve a userfriendly. An adequate accuracy compared to a traditional and digital thermometer based on IoT sensors is studied according to different distances [10]. The precise architecture design and methodologies are improved in this experimental research.

In this experimental study, the PIR sensor primarily senses motion to control the automatic light system. The sensor can detect low-frequency sensor signals according to environmental conditions, including ambient temperature and light sources whether a human moved in or out of the sensor range [11]. The sensor features temperature detection, relay indication option, hardware self-test, LED indicators, sensitivity selection, and debugging features. The applications of the PIR sensors are helpful in many areas, such as security systems, automatic lighting systems, and automatic door openers. The limitations of the PIR sensor are investigated by [12]. The sensor gives a light radiating from objects in their particular environments. It admits the energy released from things in radiation forms. The security system can vary up to detect for 5 meters. A high accuracy rate of the PIR sensor represents in [13]. Experimental results have found that the accuracy of the C-PIR sensor is $100 \%$ when it detects stationary occupants for $4.0 \mathrm{~m}$. and moving occupants for up to $8.0 \mathrm{~m}$. The PIR sensor should be installed on-board at 110 angles horizontally and 90 angles vertically.

Therefore, the innovative home system should integrate with a server to communicate between users and devices. Although there are many unpaid MQTT servers, the services have limitations with complicated modifications. When the traffic overloads or the server downs, the users may not feel comfortable living as usual. Consequently, to prevent the server's problems, Message Queue Telemetry Transport (MQTT) [14] can allow devices to communicate across constrained networks to remote systems asynchronously.

In the early fields of IoT applications, the innovative home system has provided several automatic services. Conspicuously, this study focuses on utilizing IoT technology to improve automated light control in a smart home for elderly and disable people using the Mosquitto server. The computerized light system consists of the system architecture, IoT components, software, and hardware designed to support this article. This study highlights the perspective views of the PIR sensors, such as suitable angles and distances based on the automatic light controlling system of the toilet room. 


\section{Research Objectives and Questions}

By proposing an automated light system in a smart home using IoT technology, this research aims to design the workflow overview of the automated light system based on sensors, devices, components, and servers. The research questions are defined to support the objective as follows:

RQ1: How can IoT technology be designed and implemented to support the smart home for the automated light system?

RQ2: What optimally sensing ranges of the PIR motion detector should be concerned to the prototype before using them in the actual tests?

\section{IoT Operations for Automated Light Controller}

This section presents an IoT operation that should perform to control the smart home. For example, there are many rooms in the house, such as a living room, bedrooms, bathrooms, and a kitchen. Figure 1 is an example of a regular home with a one-floor design. We configure the lights in layout design. IoT operations to produce the automated light controller with IoT technology consists of portable control using mobile devices and human detection using motion control. Two different scenarios provide to support light control. The first one is for portable control that a user enables to control any light in the smart house. For example, the elderly people are alone at night. They can turn on the living room light. The second one is automatic smart light that a system can turn on/off when a sensor detects a person. We may install the PIR sensor in the toilet room.

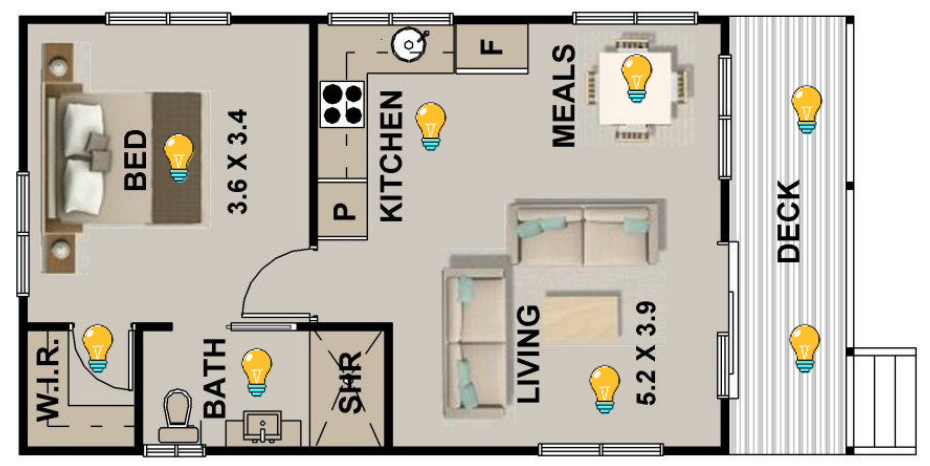

Figure 1: An example of regular home

\subsection{Portable Control}

A mobile device is a portable tool to control electrical appliances. The user can turn on and turn off the lights. There are many IoT devices in this installation, such as relay and resistors. Figure 2 shows the relay module, which is switched by an electromagnet. The web application is a web frontend using HTML/JavaScript for light control.

\subsection{Human Detection}

IoT sensors are the primary devices to sense motion. Passive Infrared (PIR) Sensor uses to detect whether a user has passed in or out of the door. Figure 3 shows the PIR sensor. In our practical study, 
we use HC-SR501 PIR motion sensor module. The sensing range is lower than 120 angles within 610 $\mathrm{cm}$. The delay time is 0.3 seconds -200 seconds. The automatic light system is developed to sense motion and provide turning on the light automatically when the sensor detects the object and turning off the light when the sensor does not detect the object.

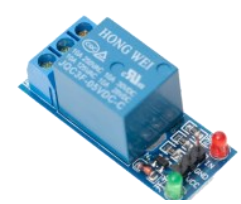

Figure 2: Relay

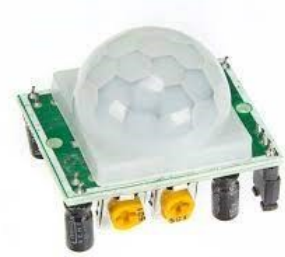

Figure 3: PIR sensor

\subsection{Diagram Outline of System}

We have demonstrated the system architecture of the automation light system design based on IoT technology (devices, software, hardware, and servers). The infrastructure architecture design of the light automation system is shown in Figure 4. A Raspberry Pi 4 is defined as a controller device that can connect to sensors, mobile devices, relays, etc., to turn on and turn off the lights both manually and automatically.

In Figure 4, the user can control the lights from the smartphone via HTTP connection. The web application displays the extensive size menu of turning on/ off the lights. The java WebSocket sends the command to the Raspberry PI 4 to switch on/off the relay. The system can repeat the commands using the sound. Moreover, the motion detection using PIR motion sensor installs in experimental rooms such as toilets or bathrooms. We install the motion sensor at the middle ceiling of experimental rooms. When the user walks into the bathroom, the sensor can detect the object and send the response to set the light on/off automatically.

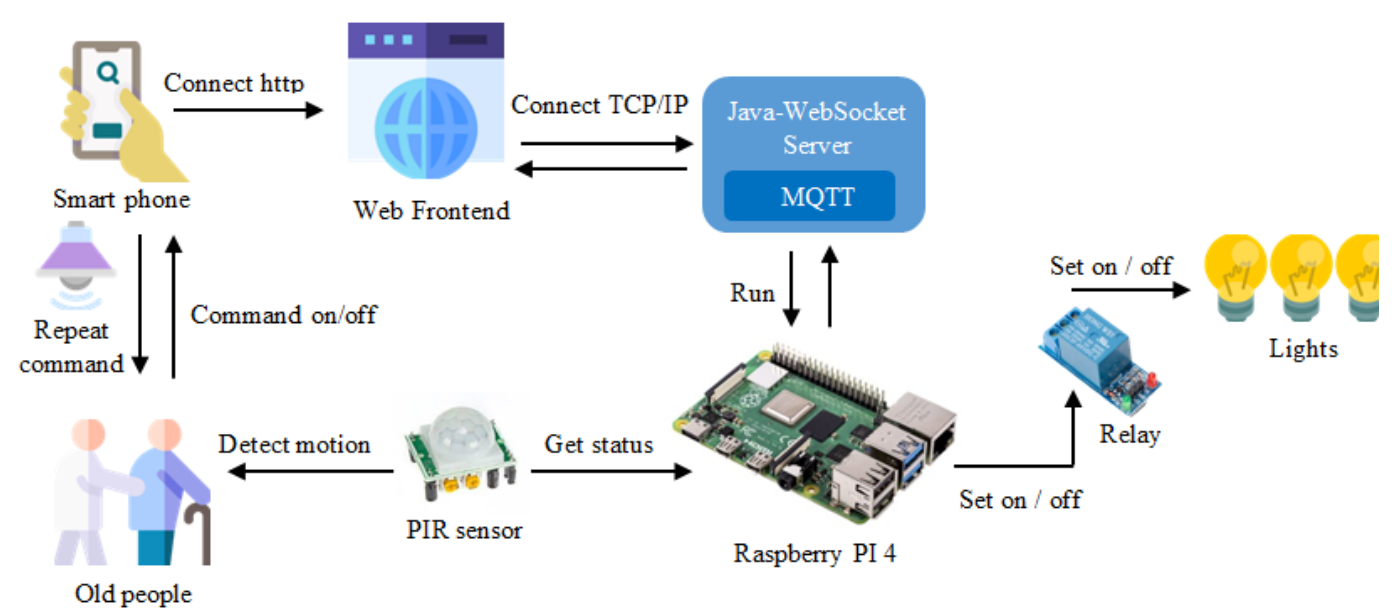

Figure 4: Diagram Outline of Automatic Light System 


\section{Implementation and Validation}

The hardware setup uses Raspberry PI 4 connecting to relay and light bulb via GPIO port by implementing the system. The software setup consists of MQTT Broker (Mosquitto on Raspberry PI 4), and JAVA WebSocket. The programming language for Raspberry PI 4 is Python. The web development languages are http and JavaScript. The concept operation of the automatic light system is shown in Figure 5. The stage starts with setting all lights off. Then, loops are handy to read the LED light status from the switch, the PIR sensor detection.

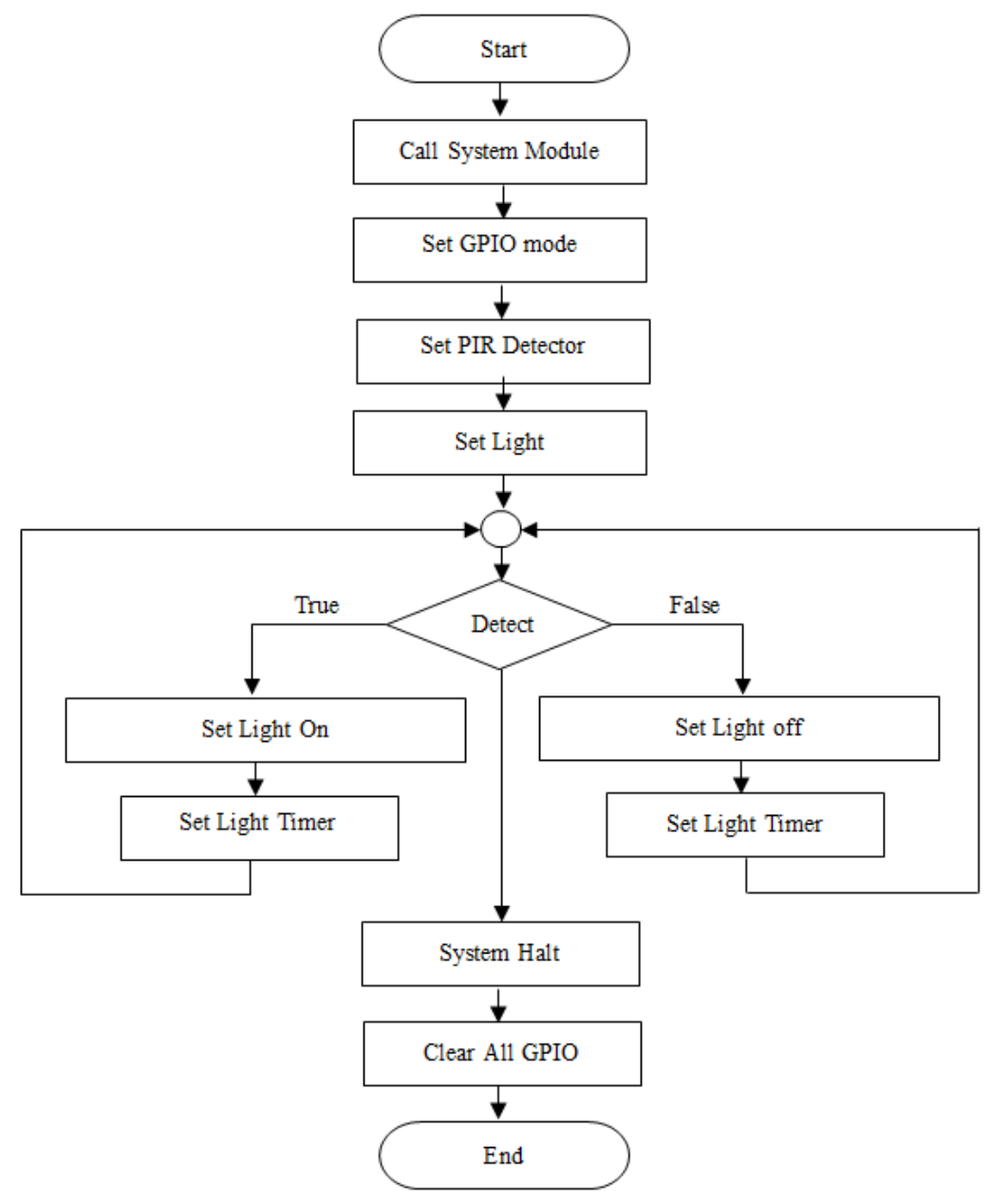

Figure 5: Concept Operation of Automatic Light System

The system validation of the system is tested to improve the software design and development. The testing cases are created to make sure that the system can deal with the test results. The system validations are light controllers to confirm that the button menu allows us to turn on/off the correct lights. The light automation of the motion sensor can prove that the light is on. When the object walks into the bathroom and the light turns off, the object walks out of the bathroom. 
Moreover, the system is easy to use according to user requirements. The user interface of the web application uses large font sizes and simple symbols that are easy to understand and recognize. The button menu can repeat the sound of the command to make sure that the user selects the right button. Network reliability using MQTT can run efficiently according to many devices connected. Table 1 shows the indication factors of functional system validation in four factors. We have found that the test methodology results can pass the standard's functional requirements.

\begin{tabular}{|c|c|c|}
\hline Test Item & Test Method & Test Result \\
\hline Light controller & $\begin{array}{l}\text { The correctness of using the menu } \\
\text { commands to turn on/off each light. }\end{array}$ & $\begin{array}{l}\text { Test results are according } \\
\text { with the standard's } \\
\text { requirement }\end{array}$ \\
\hline \multirow[t]{2}{*}{ Light Automation } & $\begin{array}{l}\text { The object walks into the bathroom, and } \\
\text { the light turns on. }\end{array}$ & Test results pass. \\
\hline & $\begin{array}{l}\text { The object walks out of the bathroom, and } \\
\text { the light turns off. }\end{array}$ & Test results pass. \\
\hline Ease of use & $\begin{array}{l}\text { The user can understand the menu using } \\
\text { symbols and the large font sizes of each } \\
\text { button. }\end{array}$ & $\begin{array}{l}\text { Test results are according } \\
\text { with the standard's } \\
\text { requirement }\end{array}$ \\
\hline & $\begin{array}{l}\text { The system can repeat the sound of } \\
\text { commands to make sure that the user } \\
\text { selects the exact button. }\end{array}$ & Test results pass. \\
\hline MQTT Reliability & $\begin{array}{l}\text { The system using MQTT can run } \\
\text { efficiently } 24 / 7 \text { while the Wi-Fi is } \\
\text { connected via other devices. }\end{array}$ & Test results pass. \\
\hline
\end{tabular}

Table 1: Indication Factors of Functional System Validation

Next, in the experimental environment of the prototype system, we have intentionally identified the sensing range of the PIR motion detector and timer to the functional system validation. Whether the PIR motion sensor works coverage as the sensor product specification described or not. We tested object movements that motion sensors can detect within 140 angles divided into 70 angles on the left and right of the middle center. The motion sensor at the central center of the ceiling is $380 \mathrm{~cm}$ from the floor. We have found that the PIR motion sensor can sense the moving object within the sensor product specification in the testing environment, such as the living room, restroom, and bedroom. As illustrated in Figure 6, the PIR motion detector can sense about 120 angles, and the sensing ranges depend on the angle of an object and the sensor. The motion sensor can detect an object up to $360 \mathrm{~cm}$ direction of 90 straight directions. The wider angles of objects upward to the left and right of the motion sensor, the closer the motion sensor can detect objects. For example, the motion sensor can detect an object at 45 angles from the center of the motion sensor on the right or left within $340 \mathrm{~cm}$. The motion sensor can provide $100 \%$ area coverage of the experimental rooms.

Additionally, we have investigated the motion timer to detecting frequency as well. The motion sensor can adjust both sensitivity detection and timer of each sense. We have found that the more sensitivity detection is set, the better or worse detection depends on the timer. When the timer of the motion sensor is set to a shorter period of sense, the motion sensor can detect with quick response. Nevertheless, the sensing coverage area is shorter distances as well. Therefore, the more sensitivity detection and shorter period timer of sense are the best results to quick response and better frequencies of motion detection. 


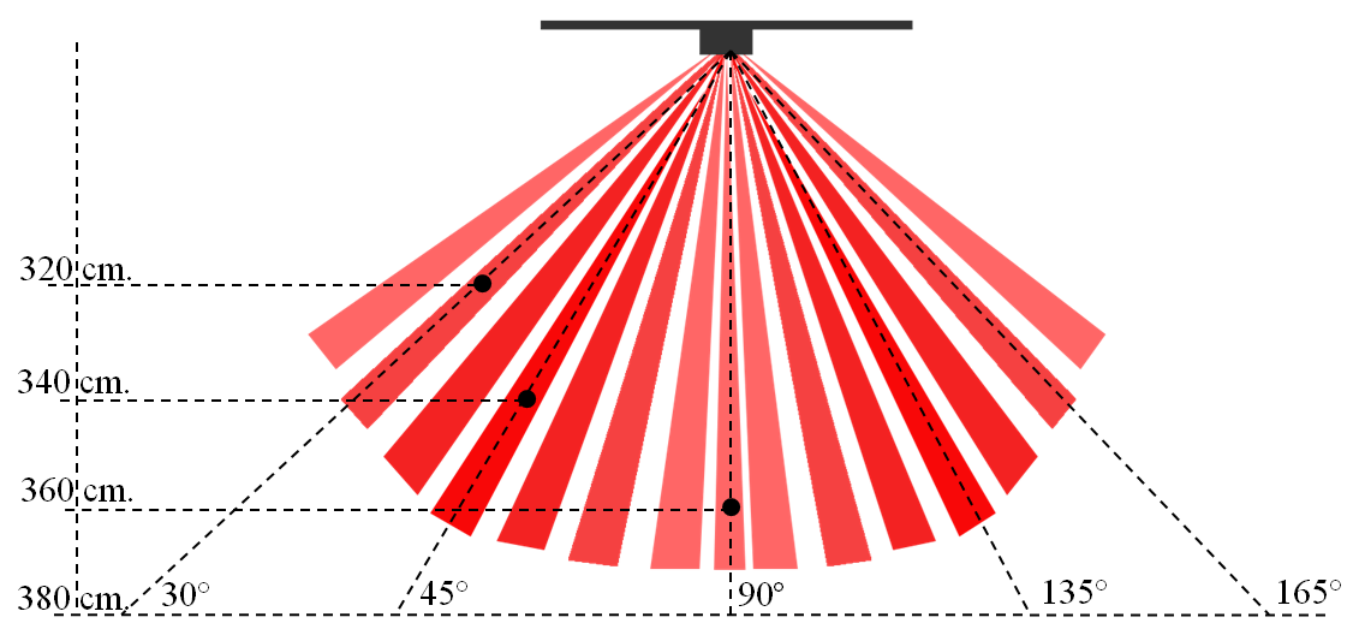

Figure 6: Distances and angels coverage areas

\section{Conclusion}

Our study responds to the system design and development of the automated light system. We have presented IoT applications based on the diagram outline of IoT sensors, devices, components, and servers. We propose an innovative home using an HC-SR501 PIR motion sensor module on Raspberry Pi 4 with an MQTT server that can perform flexibility and ability control and modify any devices in the IoT network system. The prototype design can automate a light system in a smart home using IoT technology. Our findings may inform to fulfill IoT applications for this research. For instance, there are curiosities concerns of motion detectors such as time detection of a sensor, suitable sensors for actual usage, and numbers of motion detection sensors. The motion sensor result can express a significant concern in the development. We have found that the more sensitivity detection set with, the shorter period timer of sense. It makes the system best result in quick response and better frequencies of motion detection. The wider angles of objects upward to the left and right of the motion sensor are, the closer the motion sensor can detect objects.

The automated light system can be helpful in the situation of the covid-19 disease. The system can help reduce contact between covid-19 infected persons and others and make the elderly, disabled, and blind people convenient and safe. This research can further support either sign recognition or voice command to control the light system and other things. 


\section{References}

[1] H. V. Bhatnagar, P. Kumar, S. Rawat, and T. Choudhury, "Implementation model of wi-fi based smart home system," in Proceedings of the 2018 International Conference on Advances in Computing and Communication Engineering, IEEE, 2018, pp. 23-28.

[2] A. Ghorayeb, Rob Comber, and Rachael Gooberman-Hill, "Older adults' perspectives of smart home technology: Are we developing the technology that older people want?," International Journal of Human-Computer Studies, vol. 147, pp. 102571, Novermber 2021.

[3] D. Vergados, A. Alevizos, A. Mariolis, and M. Caragiozidis, "Intelligent services for assisting independent living of elderly people at home," In Proceedings of the $1^{\text {st }}$ international conference on Pervasive Technologies Related to Assistive Environments, ACM, 2008, pp. 1-4.

[4] M. Rukhiran, P. Netinant, and T. Elrad, "Effecting of environmental conditions to accuracy rates of face recognition based on IoT solution," Journal of Current Science and Technology, vol. 10, no. 1, pp. 21-33, January - June, 2020.

[5] N. Ainane, M. Ouzzif, and K. Bouragba, "Health monitoring system in a smart home," in Proceedings of the ACM SIGGRAPH /Eurographics Symposium on Computer Animation, ACM, 2019, pp. 1-5.

[6] E. Lamiae, E.Fatiha, B.Mohammed, and G. T. Hicham, "A study on smart home for medical surveillance: Contribution to smart healthcare paradigm," in Proceedings of the $4^{\text {th }}$ International Conference on Smart City Applications, ACM, 2019, pp. 1-6.

[7] N. Chaitra, C. S. Ashwini, and S. Zaidi, "A Research on Home automation for elderly and physically challenged people by using IoT," International Journal of Innovative Technology and Exploring Engineering, vol. 8, no. 6s4, pp. 473.-475, April 2019.

[8] V. D. Vaidya, and P. Vishwakarma, "A comparative analysis on smart home system to control, monitor and securehome, based on technologies like GSM, IoT, Bluetooth and PIC microcontroller with ZigBee modulation," in Proceedings of the International Conference on Smart City and Emerging Technology, IEEE, 2018, pp. 1-4.

[9] M. Rukhiran, and P. Netinant, "'IoT architecture based oninformation flow diagram for vermiculture smart farming kit," TEM Journal, vol. 9, no. 4, pp. 1330-1337, November 2020.

[10] P. Netinant, P. Vasprasert, and M. Rukhiran, " Evaluations of effective on LWIR micro thermal camera IoT and digital thermometer for human body temperatures," in Proceedings of the 5th International Conference on E-Commerce, E-Business and E-Government, ACM, 2021, pp. 2024.

[11] L. Ada, "PIR Motion Sensor" From https://cdn-learn.adafruit.com/downloads/pdf/pir-passiveinfrared-proximity-motion-sensor.pdf.

[12] S. Umamaheswari, "PIR sensor based security system, "Annals of Robotics and Automation, vol. 4, no. 1 pp. 22-24, December 2020.

[13] H. Liu, Y. Wang, K. Wang, and H. Lin, "Turning a pyroelectric infrared motion sensor into a high-accuracy presence detector by using a narrow semi-transparent chopper," Applied Physics Letters, vol.111, pp. 243901, December 2017.

[14] L. K. McLellan, and D. A. Hunstad, "Urinary tract infection: Pathogenesis and outlook," Trends in Molecular Medicine, vol. 22, no. 11, pp. 946-957, November 2016. 